\title{
VIABILIDAD Y OPERATIVA EN LA DIGITALIZACIÓN DE CASOS RUPESTRES PREHISTÓRICO DEL “ARTE SUREÑO”: RECETAS DIGITALES DESDE TÉCNICAS SFM Y PLATAFORMAS OPEN SOURCE
}

\section{OPERATIONAL FEASIBILITY AND DIGITIZATION PROCESS OF PREHISTORIC ROCK ART IN "ARTE SUREÑO": DIGITAL GUIDELINES FROM SFM TECHIQUES AND OPEN SOURCE PLATFORMS}

\author{
Alfredo M. Maximiano Castillejo ${ }^{a,{ }^{,}}$, Camilo Barcia García ${ }^{b}$ \\ ${ }^{a}$ LaQU; Universidad Autónoma de Barcelona. Spain. g4amaximiano@gmail.com

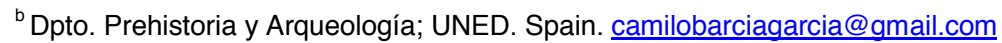

\begin{abstract}
:
We present a case study on digitalization of Southern Rock Art ("Arte Sureño") in a prehistoric rock shelter from Los Barrios, Cádiz (southern Iberian Peninsula); there we use SfM techniques to obtain some digital products for both analytic and divulgation goals. This way, we highlight some opportunities that low-cost devices (e.g. no-professional digital camera) and open software have introduced in archaeology today. We made it to converge in a working process to register and manage rock art expressions and their immediate surroundings (i.e. natural stone blocks, walls, niches...).

From this action, we not only support our work in digital 3D about multiple and varied entities that make up the scenario and neighbour context of rock art through the use of multiple low cost / free resources, but also, we intend to adjust all this operative for the processes of investigation, protection and diffusion on the varied cases that make up this unique singular artistic heritage.
\end{abstract}

Key words: Arte Sureño, Photogrammetry, Open Source, 3D Modelling

\section{Resumen:}

Presentamos el proceso de digitalización de arte rupestre prehistórico de un caso de estudio, ubicado en uno de los abrigos pertenecientes a un complejo rupestre singular de la Peninsula lbérica (Los Barrios, Cádiz). Para ello, empleamos técnicas SfM como medio de obtención de productos digitales analíticos y realísticos/divulgativos. Con esto, pretendemos destacar la oportunidad del uso de dispositivos de bajo coste (cámara digital no profesional), junto a software de licencia libre en un proceso de trabajo, desde el cual se documentan y gestionan las evidencias artísticas (arte rupestre) y su contexto próximo (soporte pétreo).

Desde esta actuación, no sólo sustentamos nuestro trabajo en digital 3D acerca de múltiples y variadas entidades que conforman el escenario y contexto próximo del arte rupestre mediante el uso de múltiples recursos "low cost"/libres, sino que además, pretendemos ajustar toda esta operativa para los procesos de investigación, protección y difusión sobre los variados casos que componen este singular patrimonio pictórico.

Palabras clave: Arte Sureño, Fotogrametría, Open Source, Modelos digitales 3D

\section{Introducción}

El estado del arte actual acerca de las sinergias entre recursos computacionales y la digitalización de conjuntos de evidencias pertenecientes al patrimonio histórico-artístico-arqueológico, demuestra no sólo la convergencia entre ambas esferas, sino además, la necesaria complementariedad e incidencia en el desarrollo de nuevas vías en lo relacionado a medios de captura, gestión de contenidos (databanks), contextos analíticos/interpretativos, y formas de visualizar resultados. En esta tesitura, el abanico de opciones a desarrollar desde la geomática para con los problemas patrimoniales conllevan una explosión de soluciones que cambian sustancialmente las formas de afrontar los problemas arqueológicos. Dentro de este amplio espectro, nos encontramos con entornos de solución que posibilitan un control del error en la representación de escenas 3D inferior a las micras bajo el uso de medios de captura que siendo accesibles, aún son caros y de complejo manejo (toma de datos y posproceso en laboratorio) desde operativas que hacen estrictamente necesario el trabajo de especialistas. Junto a la captura y preprocesado de nubes de puntos, también debemos considerar el software para el posproceso. Generalmente y desde la arqueología, recurrimos a soluciones comerciales que nos facilitan/guían en la gestión de contenidos, pero estos entornos de pago, en ocasiones y aún bajo licencia universitaria, son medios costosos y probablemente, (en nuestra opinión) lo que puede ser más significativo para los arqueólogos es que no seamos capaces de obtener un rendimiento óptimo

"Corresponding Author: Alfredo M. Maximiano Castillejo, g4amaximiano@gmail.com 
de dichas herramientas. Ante estas circunstancias, valoramos la opción de usar medios de captura y gestores de contenidos que aun siendo aparentemente más limitados/rudimentarios, se ajustan a los problemas que queremos resolver.

Es evidente, que tener un error de micras es mejor que un desfase en el modelaje entorno a milímetros o centímetros, pero atendiendo al tipo de problema patrimonial planteado, quizás debamos partir de la siguiente pregunta: ¿Cuál es el nivel de detalle que debemos usar para resolver nuestro problema? Creemos que la respuesta nos sorprendería, ya que ciertas problemáticas inherentes al patrimonio mueble/inmueble histórico-artístico-arqueológico no precisan de un nivel de detalle adscrito a una escala de micras.

Desde estas consideraciones, hemos puesto en marcha una trabajo sistemático ${ }^{1}$ cuyo objeto de estudio está compuesto por un registro (pre)histórico de más de 250 estaciones con arte parietal (pintura y grabados). De tal modo que, la opción de trabajar en digital se constituye en una de las líneas principales de actuación sobre un patrimonio con múltiples problemáticas. En esta propuesta queremos destacar dos: $l$ ) emplazamiento, ya muchas estaciones se localizan en el medio natural con muy compleja/difícil accesibilidad, y ii) casos con una precaria conservación en una clara situación de riesgo de pérdida absoluta de los conjuntos artísticos, debido a ciertos factores de degradación natural, pero especialmente por causas antrópicas (vandalismo).

En este artículo tratamos con un caso de estudio concreto, el Abrigo I/ del Conjunto de Abrigos rupestres de Bacinete (Los Barrios, Cádiz), desarrollando aspectos de captura, gestión y tratamiento analítico de datos, integrados en un flujo de trabajo (workflow) basado en datos procedentes desde SfM, y posprocesado (gestión de nubes de puntos y modelaje 3D) desde software libre de código abierto.

\section{Bacinete II: Digitalizar como medio y no como finalidad}

Partimos de la premisa de que la digitalización del patrimonio es una oportunidad en aras a mostrar, reproducir, explicar y estudiar fenómenos del pasado que de otro modo no serían observables. Así, poniendo de manifiesto sus fortalezas, la digitalización en Bacinete II aportará (Maximiano and Barcia 2017; en prensa):

Una tecnología de registro fidedigna acerca de la realidad material sin contacto directo, ni incidencia sobre los conjuntos rupestres, garantizando un "riesgo cero";

Un planteamiento de registro total y contextual acerca de las evidencias artísticas y su entorno pétreo (abrigos, cuevas, covachas, paredes de roca, etc.), proporcionando un documento digital $3 \mathrm{D}$ en soporte estable, actualizado, accesible y potencialmente inmersivo (Realidad Virtual);

\footnotetext{
${ }^{1}$ Proyecto de tesis doctoral iniciado por uno de los autores (C. B. G.) desde la Universidad Nacional de Educación a Distancia (UNED). En ella, se desarrollan de manera extensa y exhaustivamente las ideas que brevemente enunciamos aquí.
}

El establecimiento de una metodología sistemática, relativamente rápida de captura y gestión de datos que posibilite, tanto la estandarización de los contenidos, como además la integración de los mismos (y sus derivados) en múltiples entornos digitales para distintos fines (investigación, protección, divulgación).

La convergencia de los tres puntos anteriores posibilitará un enriquecimiento sustancial acerca de los conjuntos artísticos del "Arte Sureño", mostrando beneficios en torno a nuevas vías en la normalización de datos (imágenes pictóricas), en lo que compete a los procesos de investigación (disponibilidad de un documento métrico, geométrico que reproduce fielmente la realidad), a la par que oferta estrategias novedosas acerca de cómo preservar (digitalmente) los conjuntos de evidencias artísticas. Además, se faculta el desarrollo de nuevos hitos de cara a la difusión, y sensibilización de este singular patrimonio hacia el gran público.

Somos conscientes de que la digitalización del patrimonio histórico-artístico debe ir más allá de una mera recreación/reproducción de objetos, entidades y conjuntos en 3D, ya que en ocasiones, se ofrece una salida gráfica con una cuidada estética, pero que puede convertir el producto virtual en un fin en sí mismo. Frente a ello, lo que hace verdaderamente útil un proceso de digitalización es que dichos productos digitales se constituyan en medios o herramientas válidas para alcanzar fines. Pero, ¿Cómo ir más allá de una recreación digital per se?

Actualmente, muchos productos digitales patrimoniales ofertan escenas virtuales que no van más allá de funciones tipo: hacer "zoom", "rotar un objeto u orbitar a su alrededor", "cambiar la visualización" (iluminación, color, representación...) o "aportar breves explicaciones" sobre una u otra parte del elemento. Frente a esto, el reto reside en el uso de recursos computacionales para facilitar las tareas de investigación (detección, identificación, clasificación, etc.), realizar análisis de múltiples variables actuando simultáneamente (big data, simulaciones, testeo de hipótesis...), y/o el empleo de realidad virtual y aumentada, orientadas al contraste de procesos, a la divulgación inmersiva y personalizada... Ciertamente, todos ellos son campos ya explorados, pero aún en el caso del Arte Sureño se encuentran poco explotados (Maximiano and Barcia 2017; en prensa). Desde el caso de Bacinete // proponemos un ajuste sobre los tipos de soluciones alcanzables, atendiendo a las problemáticas y necesidades que presenta este caso.

Bacinete /l se integra en un complejo de abrigos próximos entre sí que presentan decoración (un total de 8 casos), conocidos como: Conjunto Rupestre de Bacinete (Fig. 1). Se trata de un conjunto de bloques y afloramientos de areniscas emplazados en las estribaciones occidentales de la Sierra del Niño, sito en el Parque Natural de los Alcornocales (Los Barrios, Cádiz). Estas manifestaciones artísticas ya fueron reseñadas hace casi un siglo por Breuil and Burkitt (1929), contando desde entonces con numerosos trabajos científicos sobre el lugar. La literatura atribuye a los conjuntos artísticos representados al arte esquemático postpaleolítico y a dinámicas de sociedades complejas, cronológicamente situadas entre el Neolítico y el Calcolítico (Díaz-Andreu and Lazaric 2014; Lazarich et al. 2015). 

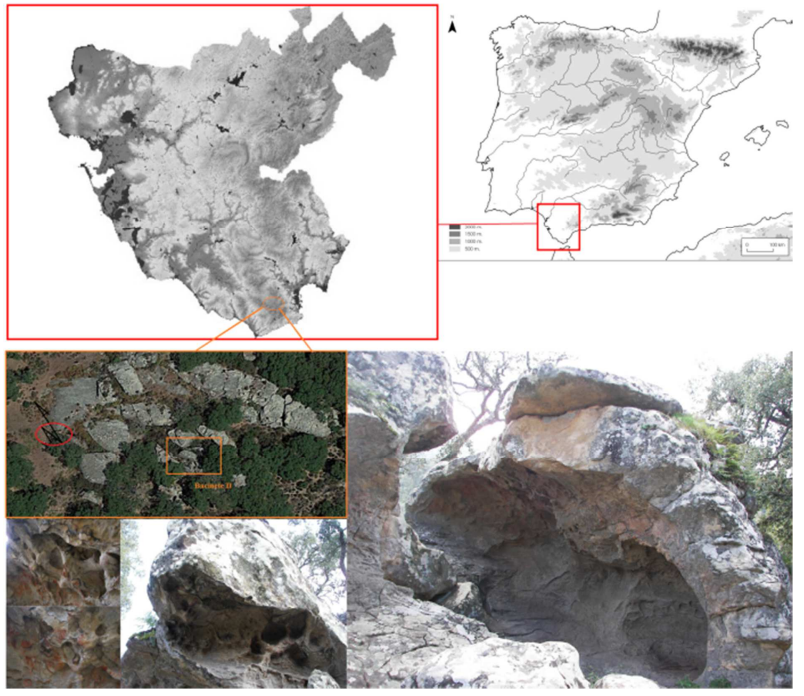

Figura 1: Localización del sitio, imagen aérea de los afloramientos y bloques rocosos. Detalles del abrigo.

Este abrigo rocoso es de pequeñas dimensiones $-2 \mathrm{~m}$. (altura) x $4 \mathrm{~m}$. (ancho) x $1 \mathrm{~m}$. (profundidad), aprox.- en el que aparecen representados diversos motivos pictóricos de tendencia no-naturalista, plasmados mediante un pigmento rojo, bastante homogéneo. Aparentemente, en esta escena no existirían grabados (Lazarich et al. 2015; Solís 2009, 2015). Las características geométricas y morfométricas del abrigo se integran en un bloque de arenisca de considerables dimensiones. El eje mayor es de unos $9 \mathrm{~m}$ de longitud (orientación E-W), y el eje menor es de unos $3.5 \mathrm{~m}$ (orientación N-S), con una altura promediada en torno a $5 \mathrm{~m}$. El abrigo y sus pinturas ocupan la cara oriental del bloque. La morfometría del mismo es compleja desde una escala global - disposición en forma pseudo tubular, con presencia de parte de cornisa y otra parte de la misma, se encuentra desprendida cuyos restos del material se encuentra desperdigado en las inmediaciones - como también, en lo referido al detalle de sus paredes, las cuales presentan numerosos entrantes y salientes.

Es muy probable, que esta configuración a escala micro debió influir en el pasado sobre la elección de los emplazamientos de las pinturas, adaptándose a los diferentes planos y concavidades existentes en el soporte pétreo. Por otra parte, destacamos las particularidades geométricas del bloque de arenisca, yendo más allá de un volumen pseudo-homogéneo en forma de hornacina y de otros tantos casos con tendencia a un plano de superficie uniforme, en los cuales aparecen los motivos artísticos. Todo ello conlleva una serie de dificultades, que son a su vez, el contexto adecuado para testear la capacidad, tanto de los medios de captura, como la gestión de los contenidos en 3D.

La digitalización de Bacinete II, como en cualquier otro caso similar, responde a unas pautas y estrategias de actuación (Fig. 2). Estas etapas secuenciadas e interdependientes deben seguir una armonización en el flujo de trabajo, el cual ha de ser viable y ágil para las capacidades de alguien versado en el tema, pero no necesariamente especialista. Por ello, estructuramos la siguiente secuencia de trabajos, con la cual pretendemos cumplir la premisa por la cual digitalizar es sólo un medio para alcanzar un fin determinado.

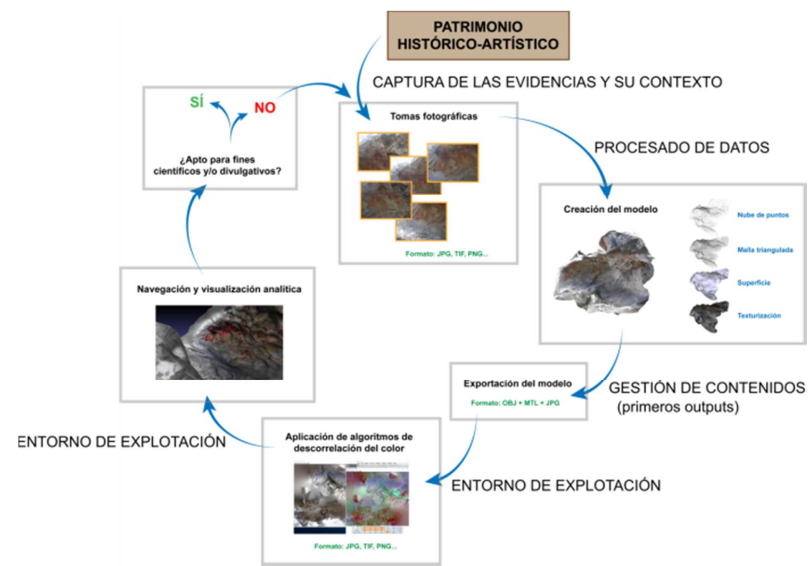

Figura 2: Esquema de trabajo de digitalización propuesto en Bacinete II.

\section{Captura de datos y gestión de contenidos: Hacia la generación de los primeros productos digitales}

La elección de la técnica de captura SfM para esta iniciativa se debe a cuatro aspectos básicos: i) Ajuste a la escala de resolución del problema planteado, ii) Metodología altamente contrastada (Agarwal et al. 2010), iii) Alta calidad, resolución y agilidad en la gestión de modelos tridimensionales de aspecto realista, y iv) Costes y accesibilidad, ya que es una técnica relativamente económica, implementable desde cámaras fotográficas digitales que cumplan con unos requerimientos mínimos, pero sin ser cámaras profesionales; y por último, disponibilidad de conjuntos de datos abordables desde diferentes soluciones de software libre. Son esos factores los que confieren a la SfM una gran versatilidad e idoneidad de cara al caso de estudio propuesto. $Y$ es con ello, con lo que podemos evaluar las potencialidades de esta metodología como sustento principal en la captura de datos para con los demás casos de estudio que planteamos abordar dentro del Arte Sureño.

Somos conscientes de que el uso de escaneo láser es la opción más precisa, pero también es cierto, que su coste económico lo hace casi prohibitivo en muchos proyectos, además de contar con un procesado de datos que es mucho más complejo y menos accesible que desde la SfM. Aunque sostenemos que la cuestión no es dirimir qué tecnología es mejor o peor, sino responder: ¿Cuál de ellas se ajusta mejor a las necesidades y expectativas reales del proyecto? Con el uso de SfM, aseguramos un control acerca del error/deformación en los modelos 3D. Atendiendo al tipo de objetivos a cubrir con esta iniciativa, hemos estimado unos valores asumibles en torno a $<6 \mathrm{~mm}$ a escala del bloque de arenisca para el estudio de las diaclasas, y de otras componentes geoformológicas; y en unos valores $<1.4 \mathrm{~mm}$ a escala de las evidencias pictóricas (a la entrega de este paper aún estamos pendiente de los resultados sobre precisión alcanzada en base a la lectura de conjuntos de puntos georreferenciados mediante Estación Total). Además, podemos acreditar la calidad geométrica de la réplica digital de los relieves de la pared rocosa (intersticios, hornacinas, entrantes y 
salientes...), y garantizamos la fidelidad de las texturas (Pereira 2013; Remondino 2014; Torres et al. 2012). Todo ello desde un entorno ágil y dinámico en torno al flujo de trabajo, ya que cada salida (output) podrá ser fácilmente exportable e incorporada (input) a otras etapas del proceso (aplicación de algoritmos de falso color, detección de bordes, caracterización de motivos, etc.).

Todo el trabajo de captura se realizó con una cámara Kodak EasyShare P850 (5,1 MP, Óptico 12x, zoom digital de 3.3x, ISO: Auto, 50 a 800, Enfoque automático: AF TTL-, único y continuo, Multi-Zone, seleccionable (25 zonas), Obturación: 16 - 1/1000 seg). Los archivos fueron capturados en formato RAW. Las tareas de procesado de datos fueron efectuadas en un primer momento a pie de campo para el contraste de la actividad, mediante un portátil HP Intel ${ }^{\circledR}$ Core $^{\mathrm{TM}}$ i7$6700 \mathrm{HQ}, 2.60 \mathrm{Ghz}$ (OS 64 bits), de RAM $16 \mathrm{~Gb}$ y una GPU Nvidia GeForce GTX960M, 4096 Mb GDDR5 (principal) e Intel® HD Graphics 530 (apoyo), con $1 \mathrm{~Tb}$ HDD + $120 \mathrm{~Gb}$ SSD de disco duro. El posprocesado y generación de modelos y demás operaciones fueron realizadas con la misma máquina desde el laboratorio.

El procesado de datos SfM fue realizado desde nubes de puntos mediante el software de libre acceso VisualSFM (Wu 2011; 2013).

Se empleó MeshLab (Cignoni et al. 2008; Callieri et al. 2013) para la restitución de geometrías, volúmenes, texturas y la georreferenciación de la modelización. Se contempla el uso de Blender para determinadas acciones (de cara a la difusión patrimonial) que a fecha de entrega de esta propuesta aún está en proceso de realización.

La estrategia de trabajo pretendía resolver tres niveles de percepción de evidencias: una aproximación global en 3D al soporte donde se encuentran la escena (bloque), definiendo en un segundo nivel el sector donde están las pinturas (abrigo) y finalmente, obteniendo los detalles concretos de las pinturas en su marco próximo (friso). Así, en una primera captura de datos alcanzamos una caracterización geométrica del todo el bloque de arenisca al completo $\left(60 \mathrm{~m}^{3}\right.$ aprox.), facilitando así la comprensión del encaje de las pinturas en su entorno, tanto dentro de todo el conjunto rupestre, cómo en el propio bloque como entidad autónoma y contenedora de las representaciones artísticas.

Para ejecutar estas capturas de datos, se realizó una baja densidad de tomas fotográficas (unas 45 ) sobre el conjunto del bloque (Fig. 3). A continuación, incrementamos la densidad de capturas centrándonos en el sector E. del bloque, donde se localiza el abrigo con pinturas (10m ${ }^{3}$ aprox.), pretendiendo recolectar el microrelieve completo de la pared que sirve de soporte al arte rupestre (Fig. 4), empleando unas 65 tomas fotográficas. Por último, llevamos a cabo un barrido exhaustivo (80 tomas) centrado en los paneles con motivos artísticos ( $4 \mathrm{~m}^{3}$ aprox.) para disponer de un documento fidedigno de las evidencias artísticas (réplica virtual) que fuera útil, tanto para procesos de investigación científica, como para el ámbito de la conservación y la divulgación de contenidos.

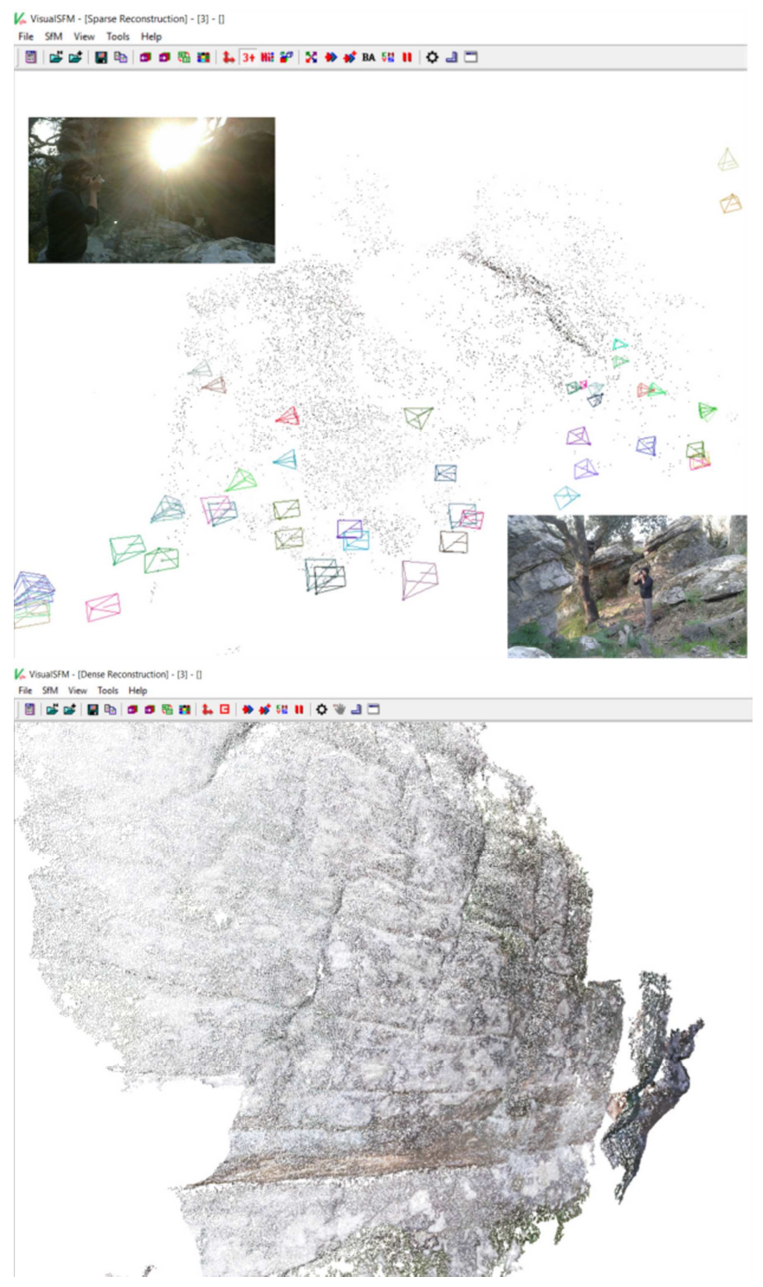

Figura 3: Toma de datos y gestión de nubes de puntos desde software libre VSfM
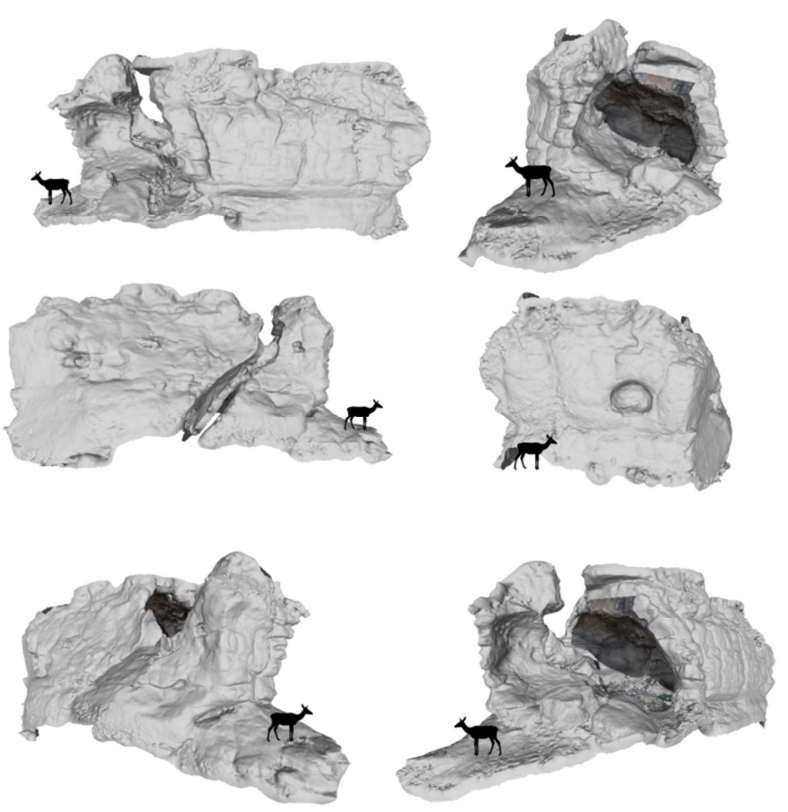

Figura 4: Modelo generado desde Meshlab, Caras orientadas del bloque en donde se encuentra el abrigo Bacinete II. (vista de abrigo en otra trama de color). Imagen tomada de Maximiano y Barcia 2017; en prensa. 
Hacemos hincapié en que los modelos generados se han centrado en los motivos artísticos y en su contexto inmediato, somos conscientes que para la restitución completa del bloque de arenisca habría sido ideal contar con apoyo técnico aéreo cercano durante el proceso de captura - UAV- nos habría facilitado mucho el registro de ciertas partes en la zona superior del bloque (tapa) y de algunos sectores de los laterales más altos del conjunto. No obstante, para la documentación detallada del abrigo y los paneles con arte, consideramos que el uso de SfM (mediante cámara digital y operado experimentado) ha alcanzado los objetivos pretendidos.

El modelo obtenido, aparte de recrear las evidencias, permite la extracción de nuevos niveles de información, como por ejemplo: métrica y volumetrías sobre cualquier elemento/s del conjunto (desde el tamaño de los motivos hasta la medida de las paredes), además posibilita cuantificar y estimar la intensidad en la degradación sobre determinadas componentes estructurales, tanto de las pinturas, como del soporte pétreo.

En lo referente a aspectos de texturas y color de los motivos pictóricos, destacamos la oportunidad que ofrecen los modelos $3 D$ y la ortofotografía en la puesta en marcha de pruebas digitales radiométricas sobre detección de falso color en torno a los tipos de pigmentos, y en la percepción de ciertas evidencias pictóricas que pueden no ser detectadas a través de las capacidades del ojo humano. Así, hemos realizado una serie de tratamientos exploratorios mediante el uso del software libre DStretch (Harman 2008; 2017) soportado desde el también software libre de procesamiento y análisis de imagen ImageJ (Abràmoff et al. 2004; Schneider et al. 2012). DStretch ha sido diseñado explícitamente para el análisis de arte rupestre, para el cual se aplican algoritmos de descorrelación sobre las longitudes de onda del espectro visible registradas en una imagen, con ello se puede descomponer el color de los píxeles, acentuando sus diferencias y confiriéndoles un falso color resaltando los matices que resultaban difusos e incluso, ocultos a simple vista (Gómar et al. 2011; Harman 2008; Quesada 2010; Martínez et al. 2013). Al aplicar los algoritmos de descorrelación y el ajuste de parámetros en DStretch directamente sobre las texturas del modelo digital 3D, y la ortofoto obtenida mediante SfM, nos permite la navegación (vía MeshLab) en la escena 3D, y percibir mejor, tanto elementos difuminados/ocultos a la vista, como la relación entre las pinturas prehistóricas en base a la morfología y el microrelieve de la pared que ha ejercido tanto de soporte, como de contexto inmediato a las pinturas.

\section{Primeros resultados alcanzados}

El principal objetivo ha sido generar una copia digital que será estable y perdurable en el tiempo, posibilitando la integración de las evidencias artísticas en cualquier tipo de Tesauro y a su vez, generar entornos de fácil interacción con dicho patrimonio sin ningún riesgo/menoscabo para el mismo (Fig. 5).

Desde el enfoque de la investigación, los productos generados permiten una parametrización en términos de métrica y geometría para con las entidades pictóricas y su contexto próximo (friso, abrigo, bloque), véase Figura 6. Además, posibilita el uso de entornos tipo visual- learning y series estadísticas sobre los conjuntos artísticos, la simulación de fenómenos físicos (Barcia and Maximiano 2015; Maximiano et al. 2015), la determinación, la estimación y la simulación de procesos de degradación y daños sobre los motivos artísticos y su contexto próximo. Junto a todo lo anterior, destacamos las opciones que se abren para la puesta en marcha de propuestas de restauración, anastilosis virtual, ya sea parcial o total, sobre casos extremadamente dañados (Grün and Remondino 2004; López 2014).

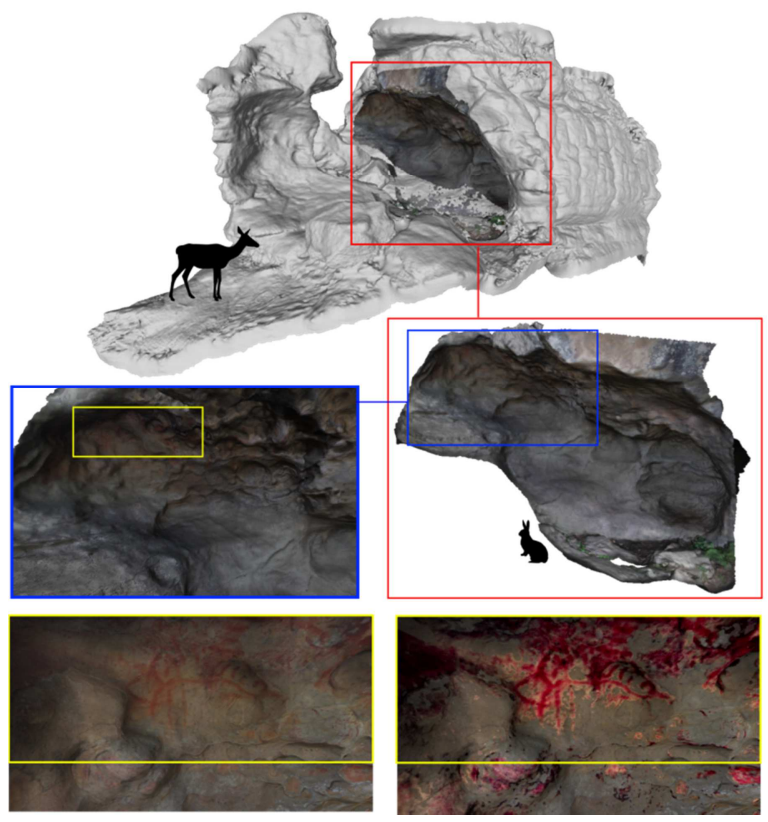

Figura 5: Visualización de las diferentes escalas de los productos 3D generados. Desde el volumen3D completo, hasta los detalles de las pinturas insertos en su correspondiente 3D (Imagen inferior derecha: filtro de color para detectar presencia de pigmentos no visibles a simple vista).

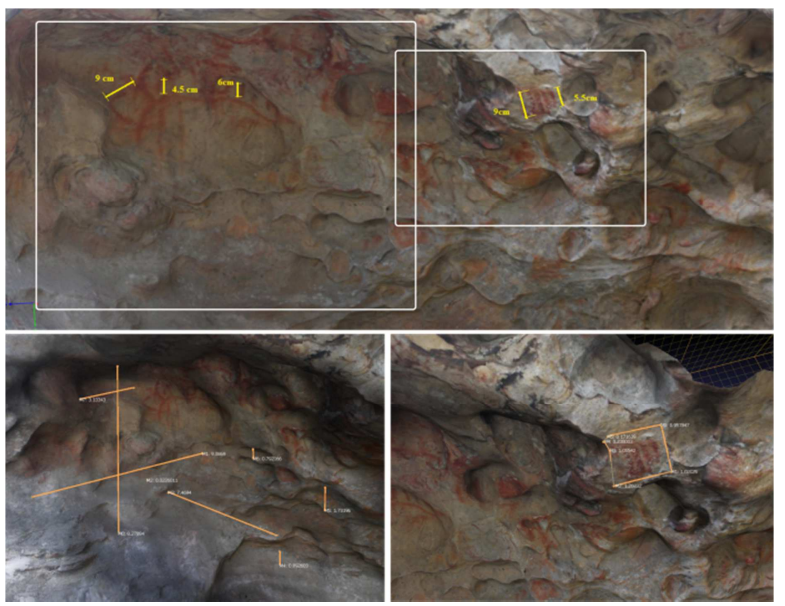

Figura 6: Ejemplos de medidas sobre algunas de las evidencias pictóricas en partes del friso.

Por otra parte, aspectos referidos a la textura y colorimetría tratados desde escenas $3 \mathrm{D}$, ortofoto y algoritmos de descorrelación de longitudes de onda (falso color) han permitido la detección de determinadas evidencias pictóricas que no son perceptible/reconocibles desde las capacidades del ojo 
humano. Estos resultados son muy interesantes, ya que permite el desarrollo de herramientas computacionales desde donde se realicen rutinas de diferentes tipos de filtros de falso color sobre paneles con arte, pudiendo contrastar la presencia de motivos pictóricos que no han sido detectados a través de los procedimientos clásicos de inspección ocular sobre dichos paneles (Fig. 7).

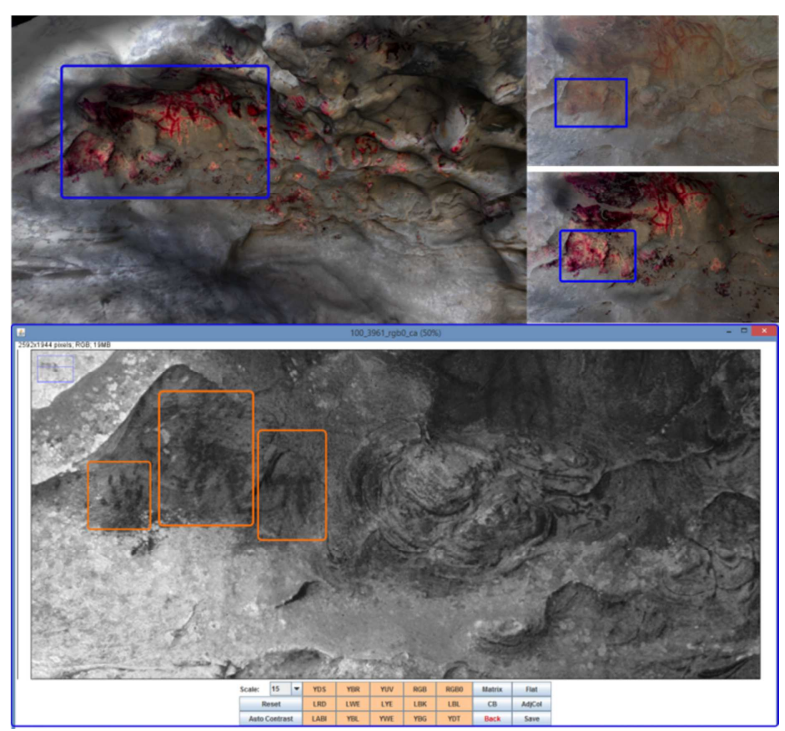

Figura 7: Parte superior de la imagen: Modelo 3D más ortofoto con algoritmo de color en donde se enfatiza los píxeles con color rojo (pigmentos) perteneciente a los motivos representados. En la parte inferior, aplicación sobre foto del algoritmo en ImageJ y Dstrech con la configuración RGBO en textura de fondo black para destacar la presencia de dos

posibles figuras que podrían representar animales cuadrúpedos $y$ algo que es indeterminado a la derecha de los mismos.

Desde una perspectiva orientada hacia las esferas de la protección y la sensibilización/difusión de este singular patrimonio artístico, los salidas digitales deben contar con una serie de peculiaridades, ya que en este caso, el arte parietal se convierte en un medio para el conocimiento de dinámicas sociales de la Prehistoria para tipos de público diferente al académico (López 2007). Con ello, se pretende aportar una infraestructura digital que permita la creación de narrativas adaptadas a diferentes tipos de colectivos de la sociedad actual, facilitando la interacción con este clúster de arte rupestre. Sin duda alguna, la mejor forma de proteger es conocer, de tal modo que la labor de difusión sobre el Arte Sureño lleva implícito un discurso adaptado a las necesidades del receptor (Maximiano y Barcia en prensa). Así, disponer de un soporte digital que se ciña a los principios de la Carta de Sevilla: International Principles Of Virtual Archaeology (http://virtualarchaeology.net/), que sea flexible y de calidad media-alta, posibilita que las acciones de sensibilización y protección sean más eficientes a través de ciertas propuestas digitales, que mediante la recurrencia a determinados soportes analógicos, los cuales siguen siendo válidos y complementarios a las soluciones digitales. En estos términos, configurar los productos alcanzados con SfM y software libre permite desarrollar contenidos en entornos como Webs inversivas, Realidad Virtual y Realidad Aumentada, contextos ideales y recomendables para que el visitante, en función de sus necesidades, pueda acceder a un discurso comprensible y útil a la hora de entender qué es lo que está viendo (e incluso, lo que ya no existe) y qué implicaciones histórico-culturales conllevan esas evidencias del pasado.

\section{Sin ánimo de concluir...}

Creemos que con el caso de estudio expuesto, encontramos una convergencia plausible entre el problema y los medios digitales que ofrecen soluciones. La técnica SfM ha permitido la generación de unos productos digitales con suficiente calidad y solvencia que, aun siendo más limitados que los resultantes mediante el uso de otras tecnologías (por ej. láserescáner), permiten alcanzar los objetivos propuestos y validar la utilidad de poner en marcha un programa de digitalización implementando recursos tecnológicos de tipo low-cost con cámaras digitales no profesionales, y el uso de software libre.

Entendemos que lo principal en una creación digital, es que sea un producto fidedigno a la realidad representada, y lo suficientemente válido que permita seguir desarrollando contenidos, atendiendo a las necesidades concretas del desarrollador y en conexión real con los potenciales usuarios. Es evidente que los productos digitales 3D son sólo copias (necesariamente imperfectas) de la realidad, algo que por sí mismo no puede reparar los daños provocados sobre muchos casos del Arte Sureño (antrópicos en su mayoría, naturales en menor medida). Ahora bien, contar con estas copias digitales constituye un paso adelante para resolver 'viejos problemas' desde nuevos enfoques y, quizás también, "democratizar" el acceso a la producción de conocimiento y desarrollar entornos más amigables que permitan un conocimiento más accesible e interactivo de estas singulares expresiones artísticas.

En definitiva, digitalizar no es un fin en sí mismo, sólo un -interesante- medio para alcanzar algunas de las pretensiones que hemos incluido en este trabajo, además de ser un catalizador de procesos en torno a la tarea de investigadores y divulgadores.

\section{References}

ABRÀMOFF, M.D., MAGALHAES, P.J. and RAM, S.J., 2004. Image processing with ImageJ. Biophotonics International, 11(7), pp. 36-42.

AGARWAL, S., FURUKAWA, Y., SNAVELY, N., CURLESS, B., SEITZ, S. M. and SZELISKI, R., 2010. Reconstructing Rome. Computer, 43(6), pp. 40-47.

BARCIA, C. and MAXIMIANO, A.M., 2015. Interpretar la oscuridad: cuevas, arte rupestre y análisis de luz simulada mediante escenas virtuales. Virtual Archaeological Review, 6(12), pp. 5-18.

BREUIL, H. and BURKITT, M.C., 1929. Rock paintings of southern Andalusia: a description of a Neolithic and Copper Age art group. Oxford, Oxford University Press. 
CALLIERI, M., RAZUGLIA, G., DELLEPIANE, M., CIGNONI, P. and SCOPIGNO, R., 2013. Meshlab as a complete open tool for the integration of photos and colour with high-resolution 3D geometry data. En G. Earl, T. Sly, A. Chrysanthi, P. Murrieta-Flores, C. Papadopoulos, I.Romanowska y D. Wheatley (eds.), Archaeology in the Digital Era, volume II: e-Papers from the 40thCAA Conference (Southampton, 2012). Amsterdam,Amsterdam University Press: pp. 406416.

CIGNONI, P., CALLIERI, M., CORSINI, M., DELLEPIANE, M., GANOVELLI, F. and RANZUGLIA, G., 2008. MeshLab: an open-source mesh processingtool. En V. Scarano, R. de Chiara y U. Erra (eds.), Sixth Eurographics Italian Chapter Conference: pp. 129-136.

DÍAZ-ANDREU, M. and LAZARICH, M., 2014. The Sound of Rock Art. The Acoustics of the Rock Art of Southern Andalusia (Spain). Oxford Journal of Archaeology, 33(1), pp. 1-18.

GÓMAR, A.M.; RUIZ, A. and CARRERAS, A.M., 2011. Tratamiento y calco digital aplicado al estudio de los abrigos de arte rupestre de la provincia de Cádiz. Almoraima, 42, pp. 287-302.

GRÜN, A. and REMONDINO, F., 2004 .Photogrammetric reconstruction of the Great Buddha of Bamiyan, Afghanistan. The Photogrammetric Record, 19(107), pp. 177-199.

HARMAN, J., 2008. Using decorrelation stretch to enhance rock art images. Paper presented at America Rock Art Research Association annual meeting (May 28 ${ }^{\text {th }}$, 2005). Avalaible: http://www.dstretch.com/AlgorithmDescription.html [03/05,2017].

HARMAN, J., 2017. DStretch: web site for the DStretch plugin to ImageJ, a tool for the digital enhancement of pictographs. Avalaible: http://www.dstretch.com/AlgorithmDescription.html [03/05,2017].

LAZARICH, M., RAMOS-GIL, A., RUIZ, A., GÓMAR, A.M., TORRES, F. and NARVÁEZ, M., 2015. Bacinete: un escenario de arte rupestre al aire libre. Varia XII - Serie arqueológica, 24, pp. 487-533.

LÓPEZ, F.J., 2007. La infografía 3D como sistema de documentación y divulgación. In J. Morín (coord.), Primer simposio Audema: la investigación y difusión arqueopaleontológica en el marco de la iniciativa privada. Madrid, Auditores de Energía y Medioambiente: pp. 429-444.

LÓPEZ, E., 2014. Out of the box: exploring the 3D modeling potential of ancient image archives. Virtual Archaeological Review, 5(10), pp. 107-116.

MARTíNEZ, F.J., MEDINA, A.J. and SAN NICOLÁS, M., 2013. Aplicación del plugin DStretch para el programa ImageJ al estudio de las manifestaciones pictóricas del abrigo Riquelme (Murcia). Cuadernos de Arte Rupestre, 6, pp.113127.

MAXIMIANO, A. M., BARCIA, C., en prensa Patrimonio digitalizado para tod@s: Recursos Digitales para el arte rupestre prehistorico del Campo de Gibraltar. En II Jornadas de Historia del Arte Campo de Gibraltar. Instituto de Estudios Campogibraltareños. Castellar de la Frontera. Mayo 2017.

MAXIMIANO, A.M., BARCIA, C., ARIAS, P. and ONTAÑÓN, R., 2015. Luces en la oscuridad: softcomputing y arte rupestre en el sitio magdaleniense de la Galería Inferior de La Garma. En A. Maximiano y E. Cerrillo (eds.), Arqueología y Tecnologías de Información Espacial: una perspectiva ibero-americana. Oxford, Archaeopress: pp. 50-74.

PEREIRA, J.M., 2013. Modelado 3D en patrimonio cultural por técnicas de structure from motion. PH Investigación, 1, pp. 77-87.

QUESADA, E., 2010. Aplicación Dstretch del software Image-J. Avance de resultados en el Arte Rupestre de la Región de Murcia. Cuadernos de Arte Rupestre, 5, pp. 9-27.

REMONDINO, F., 2014. Photogrammetry: Basic theory. En F. Remondino y S. Campana (eds.), 3D Recording and modelling in archaeological and cultural heritage: theory and best practices. Oxford, BAR International Series 2598: pp. 63-72.

SCHNEIDER, C.A.; RASBAND, W.S. and ELICEIRI, K.W., 2012. NIH image to ImageJ: 25 years of image analysis. Nature Methods, 9, pp. 671-675.

SOLíS, M., 2009. Restauración - Reconstrucción - Recreación virtual aplicada al conjunto rupestre de Bacinete. Espacio, Tiempo y Forma (Serie I, Nueva época): Prehistoria y Arqueología, 2, pp. 127-134.

SOLís, M., 2015. La pintura rupestre en el entorno de la Laguna de la Janda: Sierra del Niño (Cádiz). Cambio cultural, arte y paisaje. Tesis doctoral, Universidad Nacional de Educación a Distancia.

TORRES, J.C., ARROYO, G., ROMO, C. and DE HARO, J., 2012. 3D Digitization using Structure from Motion. CEIG 2012: XXII Spanish Computer Graphics Conference (Jaén, Spain, September 12-14th, 2012).

WU, C., 2011. VisualSFM: A Visual Structure from Motion System. Available: http://ccwu/vsfm/ [8/15, 2012].

WU, C., 2013. Towards linear-time incremental structure from motion. Proceedings of the 2013 International Conference on $3 D$ Vision: pp. 127-134. 